\title{
Transverse modes and instabilities of a bunched beam with space charge and resistive wall impedance
}

\author{
V. Balbekov* \\ Fermi National Accelerator Laboratory, P.O. Box 500, Batavia, Illinois 60510, USA
}

(Received 21 November 2011; published 21 May 2012)

\begin{abstract}
Transverse instability of a bunched beam is investigated with synchrotron oscillations, space charge tune shift, and resistive wall wakefield taken into account. A boxcar model is used for a general analysis, and truncated Gaussian distribution is invoked for details. The beam spectrum, instability growth rate, and effects of chromaticity are studied in a wide range of parameters, both with head-tail and collective bunch interactions included. Influence of internal bunch oscillations on the collective instabilities is investigated thoroughly. Landau damping caused by the space charge tune spread is discussed, and the instability thresholds of different modes of truncated Gaussian bunch are estimated.
\end{abstract}

DOI: 10.1103/PhysRevSTAB.15.054403

PACS numbers: 29.27.Bd

\section{INTRODUCTION}

Transverse instability of a bunched beam in a ring accelerator has been considered independently by Pellegrini [1] and Sands [2] with synchrotron oscillations and some internal degrees of freedom of the bunch taken into account ("head-tail instability"). Later Sacherer has investigated the effect in depth, including dependence of the bunch eigenmodes on amplitude of synchrotron oscillations ("radial modes" [3]).

The role of the bunch space charge was studied in Refs. [4,5], where it has been shown that the space charge produced tune spread can suppress many of the head-tail modes due to Landau damping, acting on instability much like other sources of the incoherent tune spread. First results have been obtained with an assumption that the space charge tune shift is significantly less than synchrotron tune. Closer examination of the problem in Ref. [5] has led to the conclusion that almost all head-tail modes are prone to Landau damping until when the space charge tune shift is about less than the synchrotron tune. The damping vanishes when the shift becomes greater, lower eigenmodes being free from the decay first. The lowest (rigid) mode is the only universal exception from the rule being potentially unstable with any space charge. Sometimes one or two following modes hold the stability as well, depending on the bunch shape.

Joint action of the space charge and a short-range wakefield was studied in Ref. [6] where instability growth rate has been found at different bunch parameters such as its shape, length, chromaticity, etc. Transverse modes coupling instability was considered in the work as well, and

\footnotetext{
*balbekov@fnal.gov

Published by the American Physical Society under the terms of the Creative Commons Attribution 3.0 License. Further distribution of this work must maintain attribution to the author(s) and the published article's title, journal citation, and DOI.
}

it has been shown that this rigorous effect cannot be caused by negative wake like the resistive wall one.

Single-bunch instabilities were actually investigated in mentioned articles because only short-range forces have been included in the consideration. A more general theory is developed in this paper where a bunched beam with an arbitrary number of bunches is examined taking into account the space charge, intrabunch, and bunch-to-bunch interactions. Both of these interactions affect the beam eigenmodes including the instability growth rate, although one or the other can dominate in specific situations resulting in different effects. The commonly used terms such as "collective modes instability" or "head-tail instability" are treated from this particular standpoint in the paper. The presentation is focused first on resistive wall instability [7-9], although the results can be rather easily adapted for other known wakefields. The boxcar model is intensively used to get a general outlook of the problem in a wide range of parameters. A more realistic truncated Gaussian bunch is closely examined in a limiting case of low synchrotron frequency, though the opposite case of high frequency is invoked as well to estimate thresholds of Landau damping. It is assumed that the external field is linear; however, nonlinearity of the space charge field is taken into account at the analysis.

\section{BUNCHED BEAM GENERAL EQUATIONS}

We will consider a beam consisting of $J$ bunches of length $2 \theta_{0}$, uniformly distributed over the azimuth at the interval of $\Delta \theta=2 \pi / J>2 \theta_{0}$. Sometimes we will use also the normalized intrabunch longitudinal coordinate $\tau$ having a range $-1 \leq \tau \leq 1$ within each of the bunches. In the beam rest frame of reference, the relation of these global and local variables is

$$
\theta=j \Delta \theta+\theta_{0} \tau
$$

where $j$ is the bunch number. As a rule, unified symbols will be used for different presentations of some function, 
with subindex $n$ added if needed. For example, linear beam density in the range of the $j$ th bunch can be presented as

$$
f(\theta) \equiv f\left(j \Delta \theta+\theta_{0} \tau\right) \equiv f_{j}(\tau) .
$$

The conjugated longitudinal momentum $u$ will be defined to satisfy the condition $A^{2}=\tau^{2}+u^{2}$, where $A \leq 1$ is the normalized amplitude of synchrotron oscillations. This variable is proportional to the momentum deviation of the considered particle with respect to the central bunch momentum. However, the proportionality coefficient is not a factor in the circumstance.

The distribution function of the $j$ th bunch will be denoted as $F_{j}(A)$, and the bunch shape will be described by the function

$$
\rho_{j}(\tau)=\int_{-\infty}^{\infty} F_{j}[A(\tau, u)] d u
$$

whose normalization is insignificant in this point and will be specified later.

When coherent transverse oscillations occur, the beam obtains transverse displacements $X(\theta, u)$, which is a fastoscillating function depending on the point of the longitudinal phase space. However, another (slower-oscillating) variable is more convenient for the present purposes:

$$
Y(\theta, u)=X(\theta, u) \exp \left[i \theta\left(Q_{0}+\zeta\right)\right]
$$

where $Q_{0}$ is the base betatron tune without space charge included, and $\zeta=-\xi / \eta$ is the effective chromaticity with $\xi$ as the conventional chromaticity and $\eta=\alpha-1 / \gamma^{2}$ as the slippage factor. Then, with space charge tune shift $\Delta Q(\theta)$ and wakefield $W(\theta)$ included, the function $Y$ satisfies the equation in the rest frame $[5,6]$ :

$$
\begin{aligned}
& \nu Y(\theta, u)+i Q_{s} \frac{\partial Y}{\partial \phi_{s}}+\Delta Q(\theta)[Y(\theta, u)-\bar{Y}(\theta)] \\
& \quad=W(\theta) \exp \left[i \theta\left(Q_{0}+\zeta\right)\right]
\end{aligned}
$$

where $\nu=Q-Q_{0}$ is the coherent tune shift away from the base tune, and $\phi_{s}$ and $Q_{s}$ are synchrotron phase and tune. Note that the space charge tune shift should be averaged over the beam transverse section as it has been shown in Ref. [5]. Therefore its nonlinearity does not occur in Eq. (5) and does not affect the coherent oscillations.

The function $\bar{Y}(\theta)$ symbolizes an average transverse displacement of all particles positioned on azimuth $\theta$ being defined by the expression

$$
\rho(\theta) \bar{Y}(\theta)=\int_{-\infty}^{\infty} F(\theta, u) Y(\theta, u) d u
$$

(similar expression is valid for the functions $X$ and $\bar{X}$ ). The general form of the wakefield is

$$
\begin{aligned}
W(\theta)= & 2 \int_{\theta}^{\infty} \rho\left(\theta^{\prime}\right) \bar{X}\left(\theta^{\prime}\right) \exp \left[i\left(\theta^{\prime}-\theta\right) Q_{\nu}\right] q\left(\theta^{\prime}-\theta\right) d \theta^{\prime} \\
= & 2 \exp \left(-i Q_{\nu} \theta\right) \int_{\theta}^{\infty} \rho\left(\theta^{\prime}\right) \bar{Y}\left(\theta^{\prime}\right) \\
& \times \exp \left[i \theta^{\prime}(\nu-\zeta)\right] q\left(\theta^{\prime}-\theta\right) d \theta^{\prime}
\end{aligned}
$$

where the wake function $q(\theta)$ depends on the beam coupling impedance and will be specified in succeeding sections. In the case in question, it is reasonable to represent the wakefield as a sum over all bunches preceding the examined one, including all preceding turns. Then, for the $j$ th bunch, Eq. (5) obtains the form

$$
\begin{aligned}
& \nu Y_{j}(\tau, u)+i Q_{s} \frac{\partial Y_{j}}{\partial \phi_{s}}+\Delta Q_{j}(\tau)\left[Y_{j}(\tau, u)-\bar{Y}_{j}(\tau)\right] \\
& =2 \int_{\tau}^{1} \exp \left[-i \lambda \theta_{0}\left(\tau^{\prime}-\tau\right)\right] q\left[\theta_{0}\left(\tau^{\prime}-\tau\right)\right] \bar{Y}_{j}\left(\tau^{\prime}\right) \rho_{j}\left(\tau^{\prime}\right) d \tau^{\prime} \\
& \quad+2 \sum_{j^{\prime}=j+1}^{\infty} \int_{-1}^{1} \exp \left\{-i \lambda\left[j^{\prime} \Delta \theta+\theta_{0}\left(\tau^{\prime}-\tau\right)\right]\right\} \\
& \quad \times q\left[j^{\prime} \Delta \theta+\theta_{0}\left(\tau^{\prime}-\tau\right)\right] \bar{Y}_{j^{\prime}}\left(\tau^{\prime}\right) \rho_{j^{\prime}}\left(\tau^{\prime}\right) d \tau^{\prime}
\end{aligned}
$$

with $\lambda=\zeta-\nu$. Periodicity conditions following from Eq. (4) have to be added to these equations:

$$
Y_{j+J}(\tau, u)=Y_{j}(\tau, u) \exp \left[-2 \pi i\left(Q_{0}+\zeta\right)\right] .
$$

\section{Symmetric beam}

If the bunches are quite identical so that $F_{j}(A)=F(A)$, $\rho_{j}(\tau)=\rho(\tau)$, and $\Delta Q_{j}(\tau)=\Delta Q(\tau)$ independently on $j$, all solutions of series (8) with periodicity condition (9) can be presented in the form

$$
Y_{j}^{(k)}(\tau, u)=\bar{Y}^{(k)}(\tau, u) \exp \left[\frac{2 \pi i j}{J}\left(\zeta+Q_{0}-k\right)\right],
$$

where $k=1,2, \ldots, J$. These collective modes depend on internal bunch oscillations, i.e., on the head-tail modes which, in this case, satisfy the equation

$$
\begin{aligned}
\nu Y^{(k)} & +i Q_{s} \frac{\partial Y^{(k)}}{\partial \phi_{s}}+\Delta Q\left[Y^{(k)}-\bar{Y}^{(k)}\right] \\
= & 2 \int_{\tau}^{1} \exp \left[-i \lambda \theta_{0}\left(\tau^{\prime}-\tau\right)\right] q\left[\theta_{0}\left(\tau^{\prime}-\tau\right)\right] \bar{Y}^{(k)}\left(\tau^{\prime}\right) \rho\left(\tau^{\prime}\right) d \tau^{\prime} \\
& +\sum_{j=1}^{\infty} \exp (-2 \pi i j \kappa) \int_{-1}^{1} \exp \left[-i \lambda \theta_{0}\left(\tau^{\prime}-\tau\right)\right] \\
& \times q\left(\frac{2 \pi j}{J}+\theta_{0}\left(\tau^{\prime}-\tau\right)\right) \bar{Y}^{(k)}\left(\tau^{\prime}\right) \rho\left(\tau^{\prime}\right) d \tau^{\prime}
\end{aligned}
$$

with $\kappa=\left(k-Q_{0}-\nu\right) / M$. Normalization condition will be used further for the symmetric beam

$$
\int_{-1}^{1} \rho(\tau) d \tau=1
$$




\section{RESISTIVE WAKE}

Resistive wall instability has been considered first in Refs. [7,8]. Corresponding wake function $q(\theta)$ is negative and can be presented in the convenient form:

$$
q(\theta)=-q_{0} \sqrt{\frac{2 \pi}{\theta}}, \quad q_{0}=\frac{r_{0} N_{b} R_{0}^{2}}{2 \pi \gamma \beta Q_{0} b^{3}} \sqrt{\frac{\Omega_{0}}{2 \pi \sigma}}
$$

with $N_{b}$ as the number of particles per bunch, $r_{0}=e^{2} / m c^{2}$ as the classic radius of the particles, $R_{0}$ as the machine radius, $\Omega_{0}=\beta c / R_{0}$ as the beam angular velocity, $\sigma$ as the specific conductivity of the beam pipe, and $b$ as the pipe radius. Substitution of this expression into Eq. (11) results in

$$
\begin{aligned}
\nu Y^{(k)} & +i Q_{s} \frac{\partial Y^{(k)}}{\partial \phi_{s}}+\Delta Q\left[Y^{(k)}-\bar{Y}^{(k)}\right] \\
= & -2 q_{0} \sqrt{\frac{2 \pi}{\theta_{0}}} \exp \left(i \lambda \theta_{0} \tau\right)\left[\int_{\tau}^{1} \frac{y\left(\tau^{\prime}\right) d \tau^{\prime}}{\sqrt{\tau^{\prime}-\tau}}\right. \\
& \left.+\sum_{j=1}^{\infty} \exp (-2 \pi i j \kappa) \int_{-1}^{1} \frac{y\left(\tau^{\prime}\right) d \tau^{\prime}}{\sqrt{T j+\tau^{\prime}-\tau}}\right]
\end{aligned}
$$

with $T=\Delta \theta / \theta_{0}$ as the bunch spacing in terms of the variable $\tau$, and the notation

$$
y(\tau)=\bar{Y}^{(k)}(\tau) \rho(\tau) \exp \left(-i \lambda \theta_{0} \tau\right)
$$

is used for shortness.

The square root in the last integral of Eq. (14) moderately depends on the addition $\left(\tau^{\prime}-\tau\right)$, so the expansion into Taylor series can be applied resulting in

$$
\begin{aligned}
& \nu Y^{(k)}+i Q_{s} \frac{\partial Y^{(k)}}{\partial \phi_{s}}+\Delta Q\left[Y^{(k)}-\bar{Y}^{(k)}\right] \\
& \simeq q_{0} \exp \left(i \lambda \theta_{0} \tau\right)\left[-2 \sqrt{\frac{2 \pi}{\theta_{0}}} \int_{\tau}^{1} \frac{y\left(\tau^{\prime}\right) d \tau^{\prime}}{\sqrt{\tau^{\prime}-\tau}}\right. \\
& \quad+\sqrt{M}\left(V_{1}(\kappa) \int_{-1}^{1} y\left(\tau^{\prime}\right) d \tau^{\prime}-V_{2}(\kappa) B\right. \\
& \left.\left.\quad \times \int_{-1}^{1} y\left(\tau^{\prime}\right)\left(\tau^{\prime}-\tau\right) d \tau^{\prime}\right)\right],
\end{aligned}
$$

where $B=M \theta_{0} / \pi$ is the bunch factor, and the designations

$$
\begin{aligned}
& V_{1}(\kappa)=-2 \sum_{j=1}^{\infty} \frac{\exp (-2 \pi i j \kappa)}{\sqrt{j}}, \\
& V_{2}(\kappa)=-\frac{1}{2} \sum_{j=1}^{\infty} \frac{\exp (-2 \pi i j \kappa)}{j \sqrt{j}}
\end{aligned}
$$

are used. Both of them are periodical functions of $\kappa$, one period of $V_{2}(\kappa)$ being plotted in Fig. 1. Because the function $V_{1}(\kappa)$ has singularities, it is multiplied by the aperiodic factor $\sqrt{|\kappa|}$ to have a more compact graph in the presented area. It goes without saying that the form of the plots

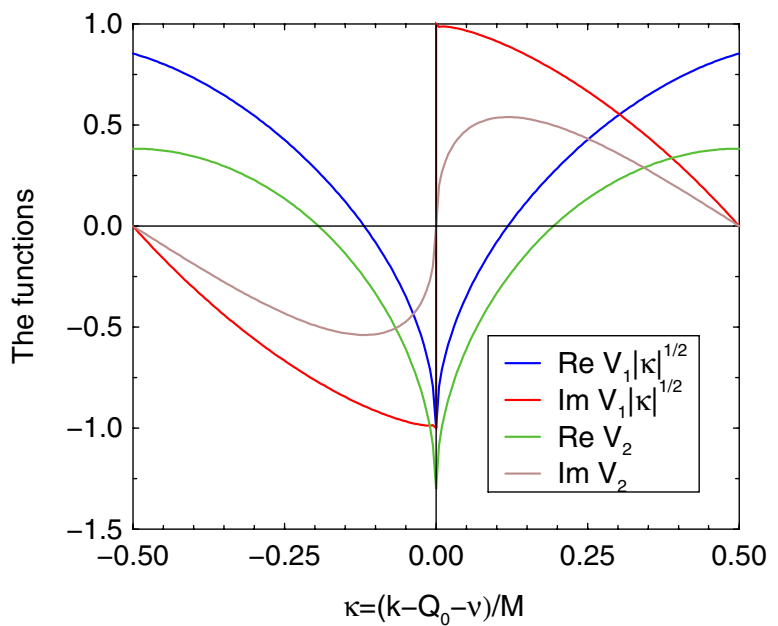

FIG. 1. Functions $V_{1}$ and $V_{2}$ characterizing the effect of resistive wall wakefield dependent on harmonic number. The functions period is $\Delta \kappa=1$, but $V_{1}(\kappa)$ is multiplied by factor $\sqrt{|k|}$ to suppress its singularity at $\kappa=0$ in the graph.

depends on wake function. However, in most cases it does not change a general structure of Eq. (15) as well as many subsequent inferences.

\section{BOXCAR MODEL}

The obtained equations can be used with any realistic distribution function, although the models with infinite linear density should be ruled out (airbag is an example). However, it may be a big problem to find their solutions, even without wakefield. In this regard, of special interest is the boxcar model, because its eigenmodes can be presented in an analytic form with $q_{0}=0$ and any $Q_{s}$ and $\Delta Q$. It gives a great possibility to outline an overall picture of the wakefield produced effects in a wide range of parameters. Therefore we consider this case in detail applying the distribution function,

$$
F(A)=F_{b c}(A)=\frac{1}{2 \pi \sqrt{1-A^{2}}}, \quad \text { at } A<1,
$$

and constant bunch density $\rho_{b c}=1 / 2$ at $|\tau|<1$, accordingly. The eigenmodes of such a bunch without wakefield (i.e., at $q_{0}=0$ ) has been investigated in Refs. [3,5]. The most important features of them are described below to be developed further with the wakefield added.

All mentioned solutions are derivable from Legendre polynomials $\bar{Y}(\tau)=P_{n}(\tau)$ with $n=0,1,2, \ldots$ Being substituted to Eq. (15) with $q_{0}=0$ and any $k$, the function $P_{n}(\tau)$ generates $2 n+1$ different solutions $Y(\tau, u)=$ $P_{n, m}(\tau, u)$ with $m=n, n-2, \ldots, 2-n,-n$. All the solutions are polynomials of power $n$ in space $(\tau, u)$ satisfying the orthogonality conditions,

$$
\iint F_{b c} P_{n_{1}, m_{1}} P_{n_{2}, m_{2}}^{*} d \tau d u \propto \delta_{n_{1}, n_{2}} \delta_{m_{1}, m_{2}},
$$




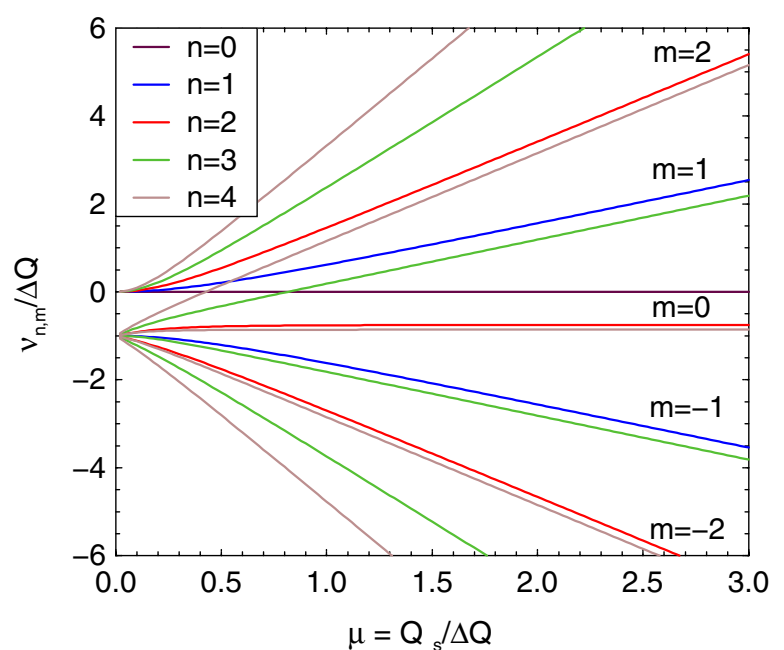

FIG. 2. Eigentunes of the boxcar bunch against synchrotron tune. Space charge tune shift $\Delta Q$ is used as a scaling factor. Two families of the tunes, starting from the points 0 and -1 at $\mu=0$, unite into multipoles at large $\mu$.

where the star symbolizes complex conjugation (similar expressions are valid with any distribution function and corresponding eigenfunctions, see [5]). Related eigentunes $\nu_{n, m}$ are real numbers depending on the parameter $\mu=$ $Q_{s} / \Delta Q$ as it is plotted in Fig. 2. It is seen that, at $\mu=0$, all eigentunes of the type $\nu_{n, n}$ start from the point $\nu=0$. It means that corresponding coherent tunes coincide with the base betatron tune $Q=Q_{0}$. All other eigentunes $\nu_{n, m<n}$ start from the point $\nu=-\Delta Q$ which corresponds to the incoherent betatron tune with the space charge included: $Q=Q_{0}-\Delta Q$. At $\mu \ll 1$, all the eigentunes acquire additions $\delta \nu \propto Q_{s}^{2} / \Delta Q$, and corresponding eigenfunctions retain about linear polarization. In this case, the functions $P_{n, n}$ depend mostly on the longitudinal coordinate $\tau$, whereas other functions $P_{n, m}$ with $m<n$ depend mostly on the momentum $u$ (numerous examples can be found in Ref. [5]). However, at $\mu \gg 1$ both kinds of solutions merge together forming the multipoles $P_{n, m}=R_{n, m}(A) \times$ $\exp \left(i m \phi_{s}\right)$ characterized by specific dependence on the synchrotron phase. The radial modes $R_{n, m}(A)$ describe dependence of the solutions on synchrotron amplitude and come from the Legendre polynomials of powers $n=$ $|m|,|m|+2$, etc. Note that the functions $R_{m, m}(A)$ represent the lowest (minimally oscillating) radial modes of given multipolarity $m$. Corresponding eigentunes are $m(m+$ $1) / 2 \times Q_{s}^{2} / \Delta Q$ at $\mu \ll 1$ and about $m Q_{s}$ at $\mu \gg 1$. The functions $R_{n, m}$ with $n>m$ have more radial oscillations being traditionally treated as higher radial modes.

\section{Low wake}

The study of the boxcar model is continued in this subsection with an additional assumption that the wakefield is small enough to apply the perturbation methods (applicability of this approximation will be discussed in Sec. V B). Then $\lambda=\zeta$ in Eq. (15), and the additions to the eigentunes can be presented in the form

$$
\begin{aligned}
\Delta \nu_{n, m}= & q_{0} \Lambda_{n, m}(\mu)\left[2 \sqrt{\frac{2 \pi}{\theta_{0}}} f_{n}\left(\theta_{0} \zeta\right)+\sqrt{M}\left[g_{n}\left(\theta_{0} \zeta\right) V_{1}(\kappa)\right.\right. \\
& \left.\left.+i h_{n}\left(\theta_{0} \zeta\right) V_{2}(\kappa) B\right]\right]
\end{aligned}
$$

with the coefficients

$$
\begin{gathered}
\Lambda_{n, m}(\mu)=\left[\iint F_{b c}\left|P_{n, m}\right|^{2} d \tau d u\right]^{-1} \\
f_{n}\left(\theta_{0} \zeta\right)=-\int_{-1}^{1} y_{n}^{*}(\tau) d \tau \int_{\tau}^{1} \frac{y\left(\tau^{\prime}\right) d \tau^{\prime}}{\sqrt{\tau^{\prime}-\tau}} \\
g_{n}\left(\theta_{0} \zeta\right)=\left|\int_{-1}^{1} y_{n}(\tau) d \tau\right|^{2} \\
h_{n}\left(\theta_{0} \zeta\right)=2 \operatorname{Im} \int_{-1}^{1} y_{n}^{*}(\tau) \tau d \tau \int_{-1}^{1} y_{n}\left(\tau^{\prime}\right) d \tau^{\prime} .
\end{gathered}
$$

In principle, these relations are adaptable to any form of the bunch with low wakefield. However, the boxcar model allows one to investigate the problem completely because its eigenfunctions $P_{m, n}(\tau, u)$ and $y_{n}(\tau)=P_{n}(\tau) \rho(\tau) \times$ $\exp \left(-i \theta_{0} \zeta \tau\right)$ are really known with any $\mu$. The results are presented graphically in Figs. 3-7 and commented below.

The coefficients $\Lambda_{n, m}$ depend only on the ratio $\mu=$ $Q_{s} / \Delta Q$ being unaffected by the bunch length and chromaticity (Fig. 3). They describe the most general action of synchrotron oscillations and space charge on transverse coherent motion of bunched beams, including the instability growth rate. It is seen that the space charge enhances the

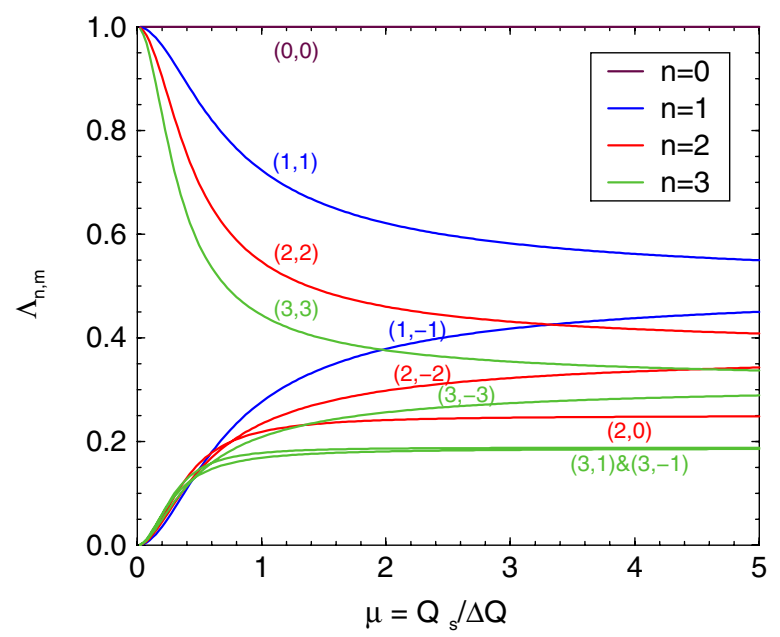

FIG. 3. Coefficients $\Lambda_{n, m}$ describing influence of synchrotron oscillations and space charge tune shift on coherent oscillations of the boxcar bunch. The eigenmode characteristic numbers $(n, m)$ are marked near the curves. 


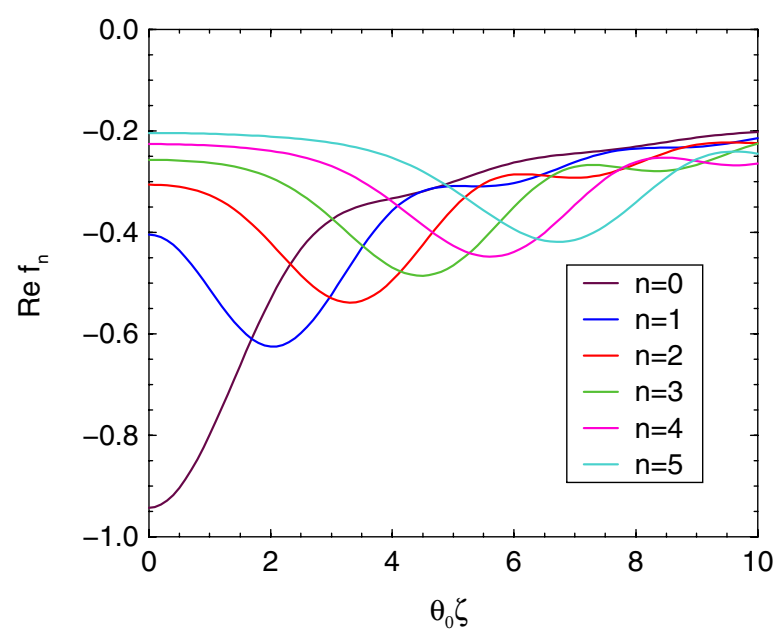

FIG. 4. Real parts of the functions $f_{n}\left(\theta_{0} \zeta\right)$ of the boxcar bunch against phase advance due to chromaticity. The curves represent contribution of intrabunch (head-tail) interaction to the coherent tune as it is described by Eq. (20). The right-hand parts of these even functions are shown.

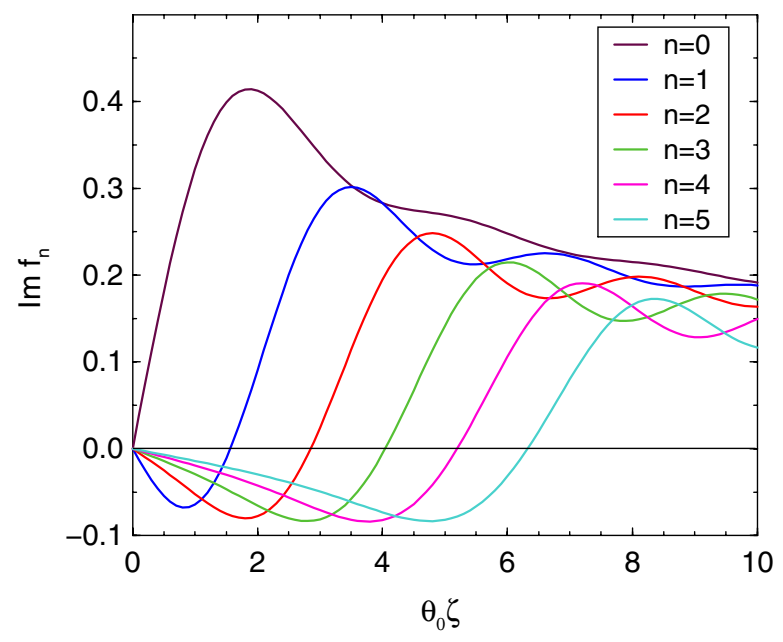

FIG. 5. Imaginary parts of the functions $f_{n}$ are plotted likewise in Fig. 4. They represent contribution of the intrabunch interaction to the instability growth rate as it is described by Eq. (20). The right-hand parts of these odd functions are shown.

effect of the wakefield on the lowest radial modes $P_{m, m}$ $(m \neq 0)$ but depresses its impact on the higher modes $P_{n, m}$ $(n>m)$. It appears well explicable taking into account behavior of different eigenfunctions at small $\mu$. As it has been mentioned above, the lowest radial functions depend only slightly on $u$, so that the overall displacement of the beam at given $\tau$ is about the same as displacements of its parts with different momentum, as it follows from Eq. (6). In contrast, the higher radial functions depend mostly on $u$ which circumstance results in some suppression of the overall beam displacement in comparison with the partial ones. Accordingly, a relative lower wakefield is excited by the higher modes, proportionally less affecting the beam

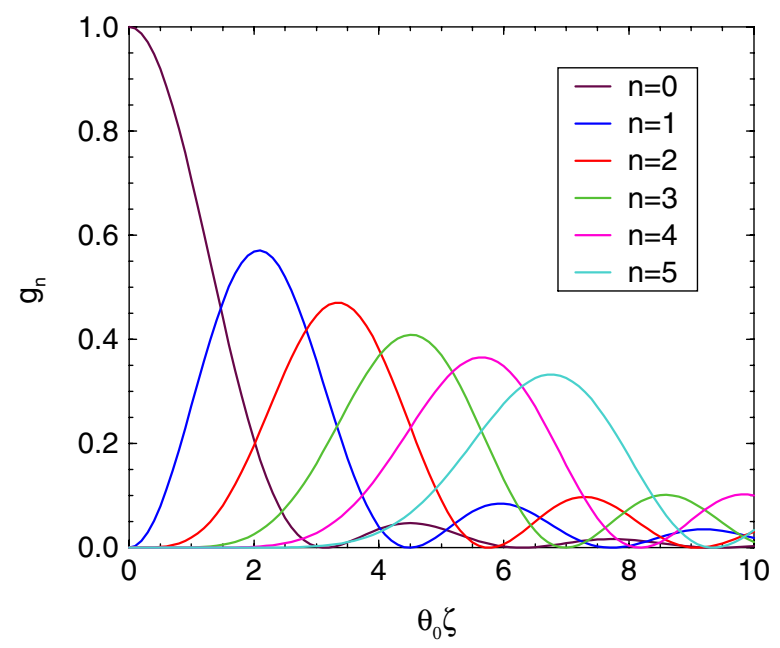

FIG. 6. Functions $g_{n}\left(\theta_{0} \zeta\right)$ of the boxcar bunch vs the phase advance due to chromaticity. They represent the major multibunch impact on coherent tunes, as it is described by Eq. (20). Only the right-hand parts of these even functions are shown.

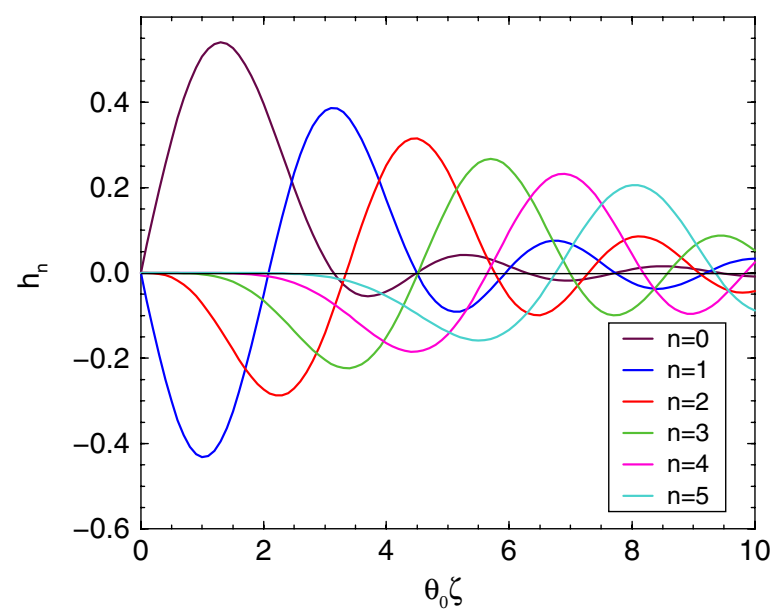

FIG. 7. Functions $h_{n}\left(\theta_{0} \zeta\right)$ of the boxcar bunch against chromaticity. The curves represent the multibunch effect due to variation of the resistive wall wakefield inside a bunch. The right-hand parts of these odd functions are shown.

motion. The important expression $\Lambda_{0,0}=1$ should be emphasized as well since it is valid with any $\mu$ and means that the space charge does not affect the lowest (rigid) mode at all. Actually, the statement is true not only for the boxcar model but for any bunched beam, because Eq. (5) with $W=0$ has a solution $\nu=0, Y=\bar{Y}=1$ with any distribution function and $\mu$.

In contrast with this, the part of Eq. (20) enclosed in square brackets describes an influence of the bunch length and chromaticity on different $n$ modes, independently on their multipolarity $m$. The part includes three terms associated with three different physical effects.

Interaction of particles inside the bunch is described by complex coefficients $f_{n}\left(\theta_{0} \zeta\right)$ which are plotted in 
Figs. 4 and 5 for several lower $n$. With nonzero chromaticity, this interaction is capable of causing the head-tail instability of different modes whose basic properties have been predicted in the earliest works [1,2]. This is a singlebunch effect which is proportional to the bunch population and does not depend on the number of the bunches. Therefore it is the most pronounced with short-range wakes being of secondary importance effect for resistive wall instability.

The second term in the square brackets describes the main effect of collective interaction of bunches. As a rule, it gives a maximal contribution to the tune shift, especially if the beam consists of many bunches. Indeed, one can get the estimations which are applicable to the most unstable modes at $M \gg 1$ :

$$
|\kappa| \simeq\left|\frac{k-Q_{0}}{M}\right| \ll 1, \quad \text { i.e. } V_{1}(\kappa) \simeq\left(1+\frac{\kappa}{|\kappa|}\right) \frac{1}{\sqrt{|\kappa|}} \text {. }
$$

Then the total contribution of this term to the tune is

$$
\Delta \nu_{n, m} \simeq \frac{q_{0} M \Lambda_{n, m} g_{n}\left(\theta_{0} \zeta\right)}{\sqrt{\left|k-Q_{0}\right|}}\left(1+i \frac{k-Q_{0}}{\left|k-Q_{0}\right|}\right)
$$

Therefore, with Eq. (13) for $q_{0}$ used, the addition to the initial coherent frequency $\omega=\Omega_{0}\left(k-Q_{0}\right)$ can be presented in the form

$$
\Delta \omega_{n, m}=\frac{r_{0} c \beta N \delta(\omega)}{2 \pi \gamma Q_{0} b^{3}}\left(1+i \frac{\omega}{|\omega|}\right) \Lambda_{n, m} g_{n}\left(\theta_{0} \zeta\right)
$$

where $N=M N_{b}$ is the total beam intensity, and $\delta(\omega)$ is skin depth at frequency $\omega$. The first part of this formula coincides with the well-known expression for resistive wall instability of coasting beams [7,8]. The factors $\Lambda_{n, m}$ reflect the impact of bunching on different head-tail modes, as it has been described above. The last multiplier $g_{n}\left(\theta_{0} \zeta\right)$ describes the influence of chromaticity. In this regard, it is pertinent to dwell on the different character of chromatic effects in coasting and bunched beams.

In the former case, chromaticity leads to a spread of incoherent betatron frequencies whose phenomenon can cause Landau damping and result in total suppressions (prevention) of the instability. In contrast with this, all particles of a bunch have one and the same average momentum because they are equalized through synchrotron oscillations. In such conditions, chromaticity does not contribute a systematic tune spread and cannot bring about Landau damping at once. Instead, the additional slip of betatron phases of particles with respect to the coherent field affects the particle oscillations resulting in a change of the instability growth rate. As it follows from Fig. 6, the instability peak transfers from lower internal modes to higher ones when the chromaticity increases, which phenomenon looks as the most descriptive chromaticity manifestation in the boxcar model.

However, it should be taken into account that chromaticity is not a sole factor which is responsible for Landau damping. In particular, space charge tune spread itself can produce a similar effect in bunched beams. As it has been shown in Refs. [4,5], the higher internal modes are more sensitive to this kind of damping and have to be suppressed first. Therefore, it is not inconceivable that increase of chromaticity leads not only to the above-mentioned shift of the instability peaks, but also to an intensification of Landau damping and to suppression of higher head-tail modes. If so, the rigid mode could be the only exclusion from the rule because it is not prone to this kind of Landau damping. However, this problem cannot be explored in the framework of the boxcar model which ignores the space charge tune spread at all. Therefore, we postpone its detailed analysis to Sec. VI where a more realistic truncated Gaussian distribution will be invoked.

Turning back to general Eq. (20), it is pertinent to note a peculiarity of a single-bunch beam which can be unstable only in a restricted region of betatron tunes. As it follows from Fig. 1 at $M=1$, imaginary parts of the coefficients $V_{1}$ and $V_{2}$ are positive only at $0<k-Q_{0}<0.5$. It means that, without chromaticity, the instability is feasible only when betatron tune is located between half integer and next integer (e.g. $Q_{0}=0.75$ but not 0.25 ). Such a result has been obtained first in Ref. [9] for a short bunch where chromaticity was ignored by the model. However, it is apparent from Eq. (20) that it is an essential factor for a real long bunch, first of all because it triggers the head-tail interaction. For example, the single bunch with zero chromaticity is quite stable at $k-Q=-0.25, B=0.5$. However, the rigid mode becomes unstable at these parameters and $\theta_{0} \zeta=1$, obtaining the growth rate $\operatorname{Im} \Delta \nu_{0,0} \simeq(0.35-0.26) q_{0}=0.09 q_{0}$ (the first term in this expression is the head-tail contribution, and the second one reflects turn by turn interaction). Of course, chromaticity of opposite sign could prevent such a situation but higher modes instability would be enforced by this, as it follows from Fig. 6.

The last term in Eq. (20) is a part of the collective interaction which describes the field variation of preceding bunches inside the considered one (Fig. 7). The influence of this addition looks much like to the head-tail interaction whose statement can be checked by comparison of Figs. 5 and 7. However, the effect strongly depends on collective mode being proportional to the coefficient $V_{2}(\kappa)$. For the example above, its contribution to the rigid mode instability is about $-q_{0} h_{0}\left(\theta_{0} \zeta\right) / 16$ which is less than the "normal" head-tail effect in order of value. Therefore, this part of the resistive wake is negligible in practice.

\section{LOW SYNCHROTRON FREQUENCY}

The boxcar model gives a chance to get a general view of the problem, but maybe it omits some important details being not sufficiently realistic by itself. Therefore, another way of looking at the problem is developed in this section based on the approach $Q_{s} \ll \Delta Q$ which is rather 
characteristic of many proton machines. As it has been shown in previous sections and illustrated by Fig. 3, in this limiting case the space charge suppresses all the modes like $Y_{n, m}(\tau, u)$ with $m \neq n$. Therefore the following results are actually concerned only with the modes $Y_{n, n}(\tau)$ which will be denoted below simply as $Y_{n}(\tau, u)$. The equation for the space part of this function is derived in Ref. [6]. Applying it with the resistive wall wakefield presented by Eq. (13), one can get the equation

$$
\begin{aligned}
& U^{2}(\tau) \bar{Y}^{\prime \prime}(\tau)-\left[\tau+\frac{U^{2} \Delta Q \rho^{\prime}(\tau)}{(\Delta Q+\nu) \rho}\right] \bar{Y}^{\prime}(\tau)+\frac{\nu(\Delta Q+\nu)}{Q_{s}^{2}} \bar{Y} \\
& =\frac{q_{0} \Delta Q \exp \left(i \lambda \theta_{0} \tau\right)}{Q_{s}^{2}}\left[-2 \sqrt{\frac{2 \pi}{\theta_{0}}} \int_{\tau}^{1} \frac{y\left(\tau^{\prime}\right) d \tau^{\prime}}{\sqrt{\tau^{\prime}-\tau}}\right. \\
& \left.\quad+\sqrt{M}\left(V_{1} \int_{-1}^{1} y\left(\tau^{\prime}\right) d \tau^{\prime}-V_{2} B \int_{-1}^{1} y\left(\tau^{\prime}\right)\left(\tau^{\prime}-\tau\right) d \tau^{\prime}\right)\right]
\end{aligned}
$$

with

$$
U^{2}(\tau)=\frac{1}{\rho(\tau)} \int F(\tau, u) u^{2} d u
$$

As it has been shown in Refs. [5,10], eigentunes of this equation are about $\nu_{n} \sim n(n+1) Q_{s}^{2} / \Delta Q$ in order of value being similar to the boxcar case in behavior. Therefore, for not very high eigenmodes with rather small synchrotron tunes, Eq. (27) can be simplified using the approximation $|\nu| \ll \Delta Q$ and resulting in

$$
U^{2}(\tau) \bar{Y}^{\prime \prime}(\tau)=Z(\tau)
$$

where

$$
\begin{aligned}
Z(\tau)=( & \left.+\frac{U^{2} \rho^{\prime}}{\rho}\right) \bar{Y}^{\prime}-\frac{\nu \Delta Q}{Q_{s}^{2}} \bar{Y}+\frac{q_{0} \Delta Q \exp \left(i \lambda \theta_{0} \tau\right)}{Q_{s}^{2}} \\
\times & {\left[-2 \sqrt{\frac{2 \pi}{\theta_{0}}} \int_{\tau}^{1} \frac{y\left(\tau^{\prime}\right) d \tau^{\prime}}{\sqrt{\tau^{\prime}-\tau}}+\sqrt{M}\left(V_{1} \int_{-1}^{1} y\left(\tau^{\prime}\right) d \tau^{\prime}\right.\right.} \\
& \left.\left.-V_{2} B \int_{-1}^{1} y\left(\tau^{\prime}\right)\left(\tau^{\prime}-\tau\right) d \tau^{\prime}\right)\right]
\end{aligned}
$$

Boundary conditions of this equation are evident from the relation $U^{2}( \pm 1)=0$ which follows from definition (28) and will be reinforced by examples in subsequent sections. Therefore, any appropriate solution of Eq. (29) should satisfy the relations

$$
Z( \pm 1)=0 .
$$

Because Eq. (27) is linear and uniform, initial conditions $\bar{Y}(1)=1, Z(1)=0$ can be used in practice to calculate the function $Z(\tau)$ everywhere with any trial $\nu$, and to separate thereafter the eigentunes $\nu_{n}$ assuring the condition $Z(-1)=0[5,6]$. The method is especially effective with $q_{0}=0$ for determination of the bunch basic modes. In particular, it confirms that Legendre polynomials are solutions of Eq. (27) in the framework of the boxcar model. Generally, it is easy to show that the basic eigenfunctions of any bunch are regular functions satisfying the orthogonality conditions

$$
\int_{-1}^{1} \bar{Y}_{n_{1}}(\tau) \bar{Y}_{n_{2}}(\tau) \rho(\tau) d \tau=\delta_{n_{1}, n_{2}}
$$

Therefore, with enough small $q_{0}$, the additions to the eigentunes can be calculated with the help of the standard perturbation technique, which results in an expression like Eq. (20):

$$
\Delta \nu_{n}=q_{0}\left[2 \sqrt{\frac{2 \pi}{\theta_{0}}} f_{n}+\sqrt{M}\left(g_{n} V_{1}+i h_{n} V_{2} B\right)\right]
$$

with the same coefficients $V_{1}$ and $V_{2}$. Equations (22)-(24) can be used as well with appropriate eigenfunctions $y_{n}(\tau)=\bar{Y}_{n}(\tau) \rho(\tau) \exp \left(-i \theta_{0} \zeta \tau\right)$ to calculate the coefficients $f_{n}, g_{n}, h_{n}$. All the factors $\Lambda_{n, n}=1$ in this limiting case due to normalization condition (32).

\section{A. Truncated Gaussian bunch with low wake}

Equation (27) can be numerically solved with different distribution functions providing rather similar results. A truncated Gaussian bunch is considered in this subsection as the most realistic example. It is characterized by the distribution function $F(A)=F_{G}(A)$ which is

$$
F_{G}(A)=\frac{C}{2 \sqrt{2} \sigma}\left(\exp \frac{1-A^{2}}{2 \sigma^{2}}-1\right) \text { at } A \leq 1,
$$

where the normalizing coefficient $C$ depends on the rms bunch length $\sigma$. Other related functions are

$$
\rho(\tau)=C\left(\frac{\sqrt{\pi}}{2} \exp \left(T^{2}\right) \operatorname{erf}(T)-T\right) \simeq \frac{2 C T^{3}}{3}\left(1+\frac{2 T^{2}}{5}\right)
$$

and

$$
U^{2}=\sigma^{2}\left(1-\frac{2 C T^{3}}{3 \rho}\right) \simeq \frac{2 \sigma^{2} T^{2}}{5}
$$

with $T^{2}=\left(1-\tau^{2}\right) /\left(2 \sigma^{2}\right)$ (approximate expressions for $|\tau| \simeq 1$ are added).

The case $\sigma=1 / 3$ ( $3 \sigma$ truncation, $C=0.016$ ) is considered below in details. Solutions of Eqs. (29) and (30) are found numerically with $q_{0}=0$ to be substituted in Eq. (33) for the tune shifts. Linear density of the bunch and six normalized basic eigenfunctions are drawn in Fig. 8. Corresponding eigentunes have the form $\nu_{n}=\alpha_{n} Q_{s}^{2} /$ $\Delta Q_{c}$ with coefficients $\alpha_{n}$ which are also presented in the picture in the brackets [for comparison: $\alpha_{n}=n(n+1) / 2$ for the boxcar model]. Here and further, the subindex $c$ marks the bunch center. 


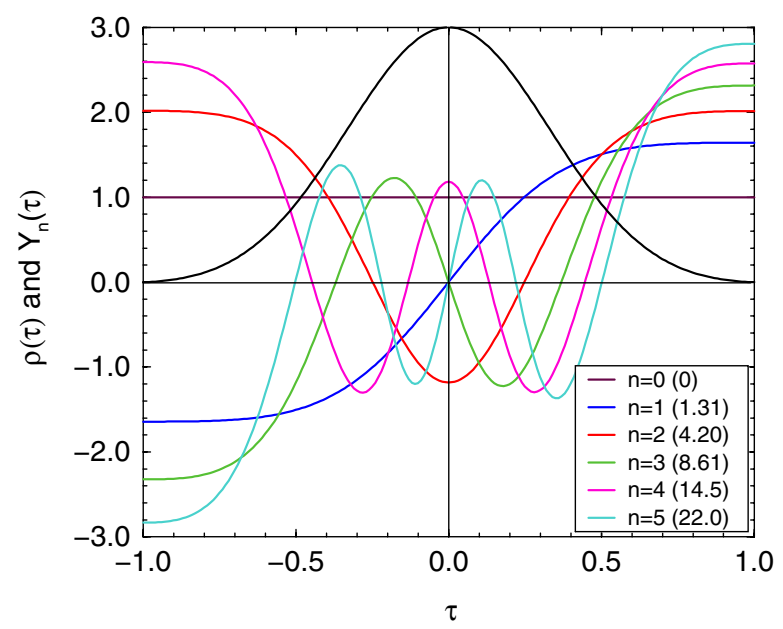

FIG. 8. Normalized eigenfunctions of Gaussian bunch truncated on the $3 \sigma$ level ( 0 th- 5 th modes). Related reduced eigennumbers $\alpha_{n}$ are given in the parentheses. The black curve represents the bunch lineal density (unnormalized).

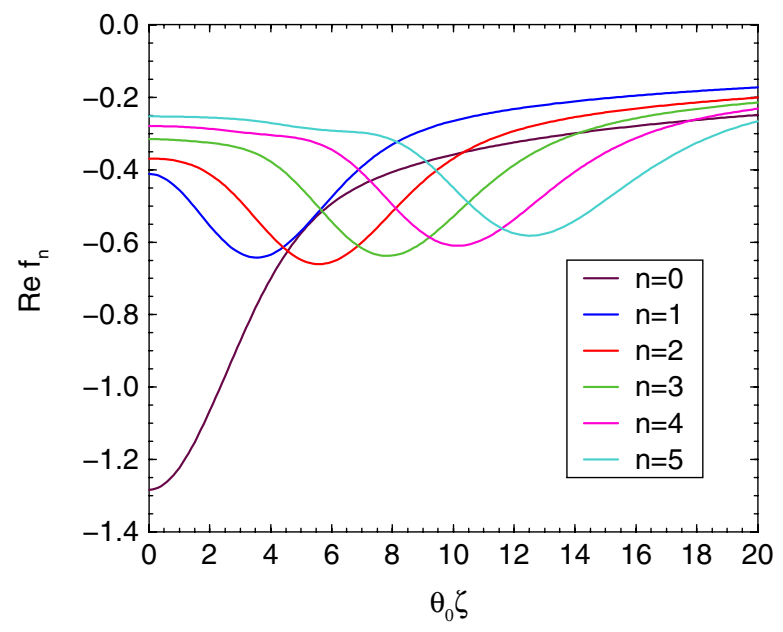

FIG. 9. Real parts of the functions $f_{n}\left(\theta_{0} \zeta\right)$ of the truncated Gaussian bunch against the bunch length and chromaticity. The curves present the contribution of intrabunch (head-tail) interaction into coherent tune, as it is described by Eq. (33). The right-hand parts of these even functions are shown.

The coefficients $f_{n}, g_{n}$, and $g_{n}$ are plotted in Figs. 9-12 which are closely similar to Figs. 4-7 associated with the boxcar model. Of course, it is necessary to take into account that the Gaussian bunch has less rms length in comparison with the boxcar one $(1 / 3$ instead $1 / \sqrt{3})$, so the Gaussian plots should be proportionally wider. An absence of the secondary oscillations is well explicable due to smoother bunch boundaries.

Other smooth models (e.g. parabolic bunch) have been checked as well and were demonstrating similar behavior if the bunch rms sizes are equalized. With these reservations, one can assert that the boxcar model provides the adequate description of bunched beam

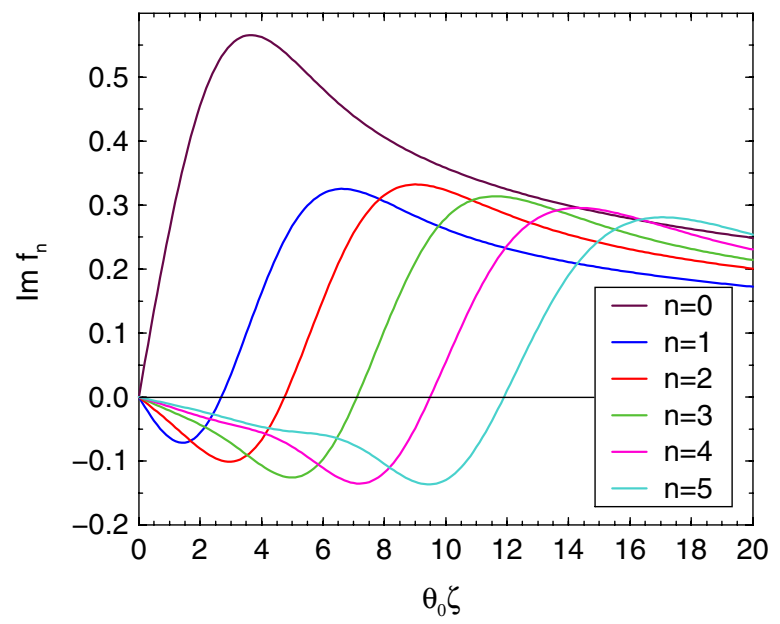

FIG. 10. Imaginary parts of the functions $f_{n}$ of the truncated Gaussian bunch are plotted likewise in Fig. 4. They represent the effect of the intrabunch interaction on the instability growth rate. The right-hand parts of these odd functions are shown.

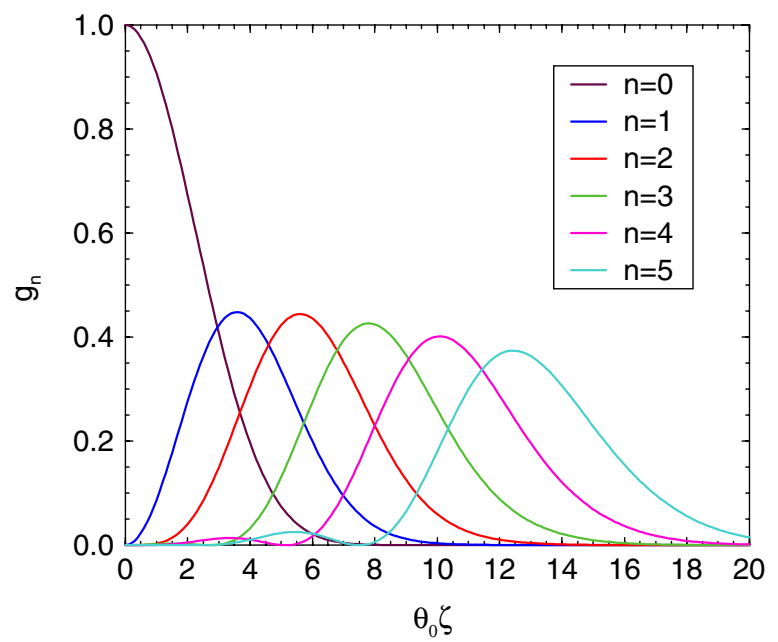

FIG. 11. Functions $g_{n}\left(\theta_{0} \zeta\right)$ of the truncated Gaussian bunch against the phase advance due to chromaticity. The curves represent the major multibunch resistive wall effect on coherent tunes, as it is described by Eq. (33). The right-hand parts of these even functions are shown.

coherent instability within the low synchrotron frequency limit.

\section{B. Expanded low wake approach}

Formally, Eq. (33) is applicable if shifts of the spectral lines $\Delta \nu_{n}$ are small in comparison with basic separations of the lines which are

$$
\nu_{n+1}-\nu_{n}=\left(\alpha_{n+1}-\alpha_{n}\right) \frac{Q_{s}^{2}}{\Delta Q_{c}} \simeq(n+1) \frac{Q_{s}^{2}}{\Delta Q_{c}} .
$$

Therefore, for the lowest mode, the condition of applicability is 


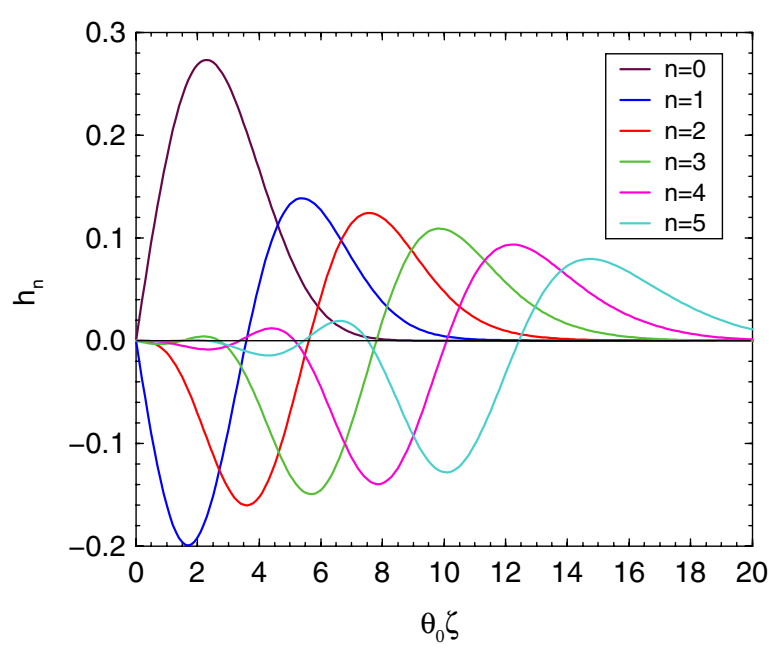

FIG. 12. Functions $h_{n}\left(\theta_{0} \zeta\right)$ of the truncated Gaussian bunch. The curves represent the multibunch effect due to variation of the resistive wall wakefield inside a bunch. The right-hand parts of these odd functions are shown.

$$
\left|\Delta \nu_{0}\right| \ll \frac{Q_{s}^{2}}{\Delta Q_{c}} .
$$

The left-hand part of this expression is close to the instability growth rate whose value is essentially less than 1 , in practice (for example, $\left|\Delta \nu_{0}\right|<0.1$ ). However, the righthand part may be still less because it includes the square of the synchrotron frequency, e.g. $\Omega_{s}^{2} / \Delta Q_{c}=0.05^{2} / 0.25=$ 0.01 . This example is provided to demonstrate that a violation of condition (38) is a quite possible occasion, especially when the collective modes instability of a multibunch beam is considered.

Therefore, we will investigate this important case in greater detail without the assumption that the multibunch contribution is extremely small. To do this, one can draw on the fact that dependence of the major part of the wakefield $\left(\propto V_{1}\right)$ on the coordinate is known, being given by the factor $\exp \left(i \lambda \theta_{0} \tau\right)$. Therefore, solutions of Eq. (29) can be presented in the form

$$
\begin{aligned}
\bar{Y}(\tau)= & q_{0} V_{1}(\kappa) \sqrt{M} \int_{-1}^{1} \bar{Y}\left(\tau^{\prime}\right) \rho\left(\tau^{\prime}\right) \\
& \times \exp \left(-i \zeta \theta_{0} \tau^{\prime}\right) d \tau^{\prime} \sum_{n=0}^{\infty} \frac{e_{n} \Upsilon_{n}(\tau)}{\nu-v_{n}},
\end{aligned}
$$

where $Y_{n}$ and $v_{n}$ are the eigenfunctions and eigentunes of the equation with $V_{1}=0$, and $e_{n}$ are coefficients of the expansion

$$
\exp \left(i \lambda \theta_{0} \tau\right)=\sum_{n=0}^{\infty} e_{n} \Upsilon_{n}(\tau)
$$

It immediately results in the dispersion equation,

$$
1=q_{0} V_{1} \sqrt{M} \sum_{n=0}^{\infty} \frac{e_{n}}{\nu-v_{n}} \int_{-1}^{1} \Upsilon_{n}(\tau) \rho(\tau) \exp \left(-i \zeta \theta_{0} \tau\right) d \tau
$$

In principle, new basic eigenfunctions and eigentunes could be found with the help of the same technique which has been used in the beginning of this section for determination of similar values $\bar{Y}_{n}(\tau)$ and $\nu_{n}$ However, it would be a more difficult problem to calculate the coefficients $e_{n}$ because the functions $\Upsilon_{n}(\tau)$ are not orthogonal, in contrast with $\bar{Y}_{n}$. Therefore we need to turn back to the approximation $Y_{n}=\bar{Y}_{n}$ which is certainly acceptable at low headtail interaction, and does not violate the overall structure of Eq. (41). As a result, the dispersion equation obtains the form

$$
1 \simeq \sum_{n=0}^{\infty} \frac{q_{0} V_{1} \sqrt{M} g_{n}\left(\theta_{0} \zeta\right)}{\nu-\nu_{n}-q_{0}\left(\sqrt{8 \pi / \theta_{0}} f_{n}+i h_{n} V_{2} B \sqrt{M}\right)}
$$

with the same coefficients $f_{n}, g_{n}, h_{n}$ which have been presented by Figs. 9-12 for the truncated Gaussian bunch. With an additional condition

$$
q_{0}\left|V_{1}\right| \sqrt{M} \ll \frac{Q_{s}^{2}}{\Delta Q_{c}},
$$

the low wakefield approximation is totally satisfied, and Eq. (42) gives the same result as earlier, Eq. (33).

Another easy but very important case is zero chromaticity, when sum (42) holds the only term $n=0$ because $g_{n}(0)=\delta_{n, 0}$. It means that solely the rigid internal mode can be excited at such conditions. Appearing inside some collective mode, it provides the tune shift,

$$
\Delta \nu_{0}=q_{0}\left[2 \sqrt{\frac{2 \pi}{\theta_{0}}} f_{0}(0)+\sqrt{M} V_{1}\left(\frac{k-Q_{0}}{M}\right)\right] .
$$

This expression formally coincides with Eq. (33) at $\zeta=0$ but can be applied with higher coefficients $V_{1}$.

Generally, series (42) contains a restricted number of summands whose conclusion follows from Figs. 6 and 11. In particular, one can see that the terms $n=0$ and $n=1$ give the major contributions to the sum at $\left|\theta_{0} \zeta\right|<\sim 3$. Equation (42) has two actual solutions in this case, at least one of them can be unstable. In the extreme case when inverse inequality of Eq. (43) is fulfilled, one of the eigentunes is real, and another frequency shift is

$$
\begin{aligned}
\Delta \omega & \simeq q_{0} \Omega_{0} V_{1}(\kappa) \sqrt{M}\left[g_{0}\left(\theta_{0} \zeta\right)+g_{1}\left(\theta_{0} \zeta\right)\right] \\
& \simeq \frac{r_{0} c \beta N \delta(\omega)}{2 \pi \gamma Q_{0} b^{3}}\left(1+i \frac{\omega}{|\omega|}\right)\left[g_{0}\left(\theta_{0} \zeta\right)+g_{1}\left(\theta_{0} \zeta\right)\right] .
\end{aligned}
$$

Rather weak dependence of this expression on chromaticity engages one's attention. In relative units, the addition is 1 at $\theta_{0} \zeta=0$ and about 0.8 at $\theta_{0} \zeta=3$. However, it should be reminded again that the low synchrotron frequency limit is considered here. The role of this factor and possible consequences of its violation are discussed in the next section. 


\section{THE INSTABILITY THRESHOLD}

It could be concluded from previous analysis that the boxcar is a quite adequate model for investigation of the bunched beam instability, and only minor and almost obvious changes are needed for more realistic distributions. However, it would be a premature conclusion because the space charge tune spread is ignored in the boxcar model. Meanwhile, in certain situations the spread can cause Landau damping and suppress many unstable modes of the real bunch, as it has been shown in Refs. [4,5]. The rigid intrabunch mode is the only occasion when this mechanism does not work and cannot prevent instability with any combination of other parameters.

At present, the problem is adequately covered only in the limits $\mu \gg 1$ and $\mu \ll 1$. In the first case, this kind of Landau damping truly works and suppresses almost all internal modes [5]. However, the mechanism is turned off in the opposite limiting case which was just the subject of the previous section. The only conclusion can be done from these facts: this stabilization mechanism has a threshold character being actually turned on at rather large $\mu$. The goal of this section is to get a more exact estimation of the threshold on the assumption that space charge makes a predominant contribution to the beam coupling impedance.

It has to be restated preliminarily that Landau damping arises when coherent frequency penetrates rather deep into the area of incoherent tunes of the system. Then the particles which own tunes are located below or above the coherent tune, are exited in contraphases by the coherent field, transforming its energy to the incoherent form (beam heating). This mechanism generates the beam decoherence and creates the instability threshold.

A well-known practical recommendation follows from this statement for coasting beams: incoherent tune spread should exceed the space charge tune shift to avoid the beam instability. In principle, a wakefield (for example, resistive wall contribution) affects this criterion; however, its influence is small in practice if the space charge dominates in the impedance budget. The last approximation is just the case of our exploration.

An additional important property of bunched beams is that, with coherent frequency $\omega$ and synchrotron one $\Omega_{s}$, the particles experience an influence of all harmonics with frequencies $\omega+l \Omega_{s}$, where $l$ is an integer. The intense energy transfer is possible if any of them falls in the region of incoherent betatron frequencies averaged over the synchrotron phase. A fast look at Fig. 2 reveals that harmonics of the index $l=m$ have the most chance to do this, with a given bunch mode. Therefore, more informative graphs can be obtained by a transformation of each curve $\nu_{n, m}(\mu)$ to the $\nu_{n, m}-m \mu$. The results are presented in Fig. 13 for the boxcar model by solid lines of the same color as in Fig. 2.

It looks like a reasonable assumption that a coherent spectrum of a realistic bunch has similar behavior, at least

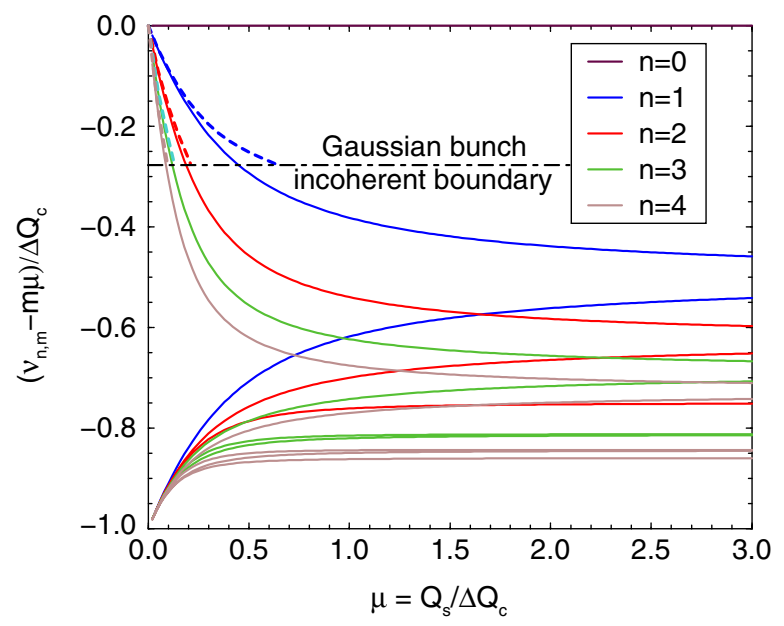

FIG. 13. Transformed eigentunes against synchrotron tune: solid lines-boxcar model; dashed lines-Gaussian bunch with $3 \sigma$ truncation. Space charge tune shift in the bunch center is used for scaling. Averaged over time incoherent betatron tunes are located below the presented incoherent boundary.

qualitatively. However, incoherent tune spread has a nonzero value in such a case, in contrast with the boxcar model. In particular, averaged tunes of the truncated Gaussian bunch as considered in Sec. VA fall in the region $-1<\nu / \Delta Q_{c}<-0.274$ which is marked in Fig. 13 by the dot-dashed line.

The following conclusions can be drawn from these plots: (i) rigid mode oscillations $(n=m=0)$ are potentially unstable with any $\mu$; (ii) lowest radial modes $(n=m)$ of higher multipoles $(m \geq 1)$ can be unstable at $\mu<\sim 1$; (iii) the more is $m$, the less is the corresponding threshold of $\mu$; (iv) all higher radial modes $n>m$ are stable with any $\mu$ because their coherent tunes start from the point $\nu=-1$ and fall into the incoherent area.

These conclusions are in a reasonable agreement with Ref. [5] where it has been shown that only the rigid mode $(0,0)$ of the Gaussian bunch is unstable at $\mu \gg 1$. One can anticipate from Fig. 13 that thresholds of other modes are arranged at $\mu<0.5$. It is a region where the low $\mu$ approximation could be fitted to refine the results. To accomplish this, we consider Eq. (27) with $q_{0}=0$ but without previously used simplification $\nu \ll \Delta Q_{c}$. In such a case, boundary conditions (31) should be a little changed to obtain the form

$$
Y^{\prime}( \pm 1)=\frac{\nu^{2}}{Q_{s}^{2}}
$$

Numerical solutions of the equation have been calculated with help of the same technique as previously. Corresponding eigentunes are plotted in Fig. 13 by dashed lines located above the Gaussian incoherent boundary because they should not penetrate in this region, as it is argued above. The merge points which can be treated as estimated thresholds of the head-tail modes are presented in Table I. 
TABLE I. Estimated instability thresholds of Gaussian bunch with $3 \sigma$ truncation (lower radial modes, no chromaticity).

\begin{tabular}{lccccc}
\hline \hline$n$ & 0 & 1 & 2 & 3 & 4 \\
\hline$Q_{s} / \Delta Q_{c}$ & $\infty$ & 0.63 & 0.21 & 0.13 & 0.095 \\
\hline \hline
\end{tabular}

Distributions with more pronounced boundaries demonstrate similar behavior but maybe a higher stability. For example, three modes of parabolic bunch retain the stability at $\mu \rightarrow \infty$ [5]. The boxcar bunch is an extreme case when all the modes are unaffected by this sort of damping. It is pertinent to note some repulsing of coherent tunes from the incoherent area whose effect enhances with a higher value of $\mu$.

Although these statements are nothing more than estimations founded on extrapolation of the limiting cases $\mu \ll 1$ and $\mu \gg 1$, nevertheless they allow one to make important suggestions concerning an influence of chromaticity on stability of bunched beams with wakefields. As it might be expected from Figs. 6 and 11, an increase of chromaticity should not drastically affect the instability growth rate, because its main effect is simply a switch of the bunch oscillations from a lower internal mode to a higher one. For example, it follows from Fig. 11 that the most unstable internal modes of the Gaussian bunch are $n=1$ at $\left|\theta_{0} \zeta\right|=4$ and $n=2$ at $\left|\theta_{0} \zeta\right|=6$, in both cases the instability growth rate being about 0.45 in used relative units (the head-tail contribution is neglected in the estimations because it is relatively small in multibunch beams). However, now we must take into account that these results were obtained with the assumption $\mu=0$. Let us consider the case $\mu=0.5$ as another example. Then, according to Table I and Fig. 11, modes $n \geq 2$ cannot be observed being suppressed by Landau damping, and chromaticity phase advance $\left|\theta_{0} \zeta\right| \simeq 10$ is practically sufficient to suppress the instability entirely, including both 0 th and 1 st modes.

\section{SUMMARY AND CONCLUSION}

Transverse instability of a bunched beam is studied in the paper with synchrotron oscillations, space charge, and wakefield taken into account. The resistive wall wakefield is considered as the most commonly encountered and practically important case. However, many of the results have a wider field of applicability and, with small changes, can be adapted to other wake functions. The boxcar model of the bunch is extensively used to reach a general outline of the problem in a vast range of parameters. A more realistic Gaussian distribution is invoked in some cases for comparison and detailed investigations of important problems including Landau damping.

Eigenfunctions and eigentunes of the beam are investigated both with intrabunch and interbunch interactions taken into account. Contributions of the interactions to the instability growth rate are studied over a wide range of the parameters, including an impact of the bunch length and chromaticity. It is shown that the wide known head-tail and collective mode instabilities are the extreme cases when one kind of the interaction certainly dominates. However, an essential influence of the intrabunch degrees of freedom on the collective instabilities is especially emphasized and investigated in detail. In particular, it is shown that a variability of the internal bunch modes explains why the instability growth rate depends on the bunch parameters including space charge tune shift, synchrotron tune, bunch length, and chromaticity.

It is noted that space charge tune spread can cause Landau damping and suppress most of the unstable modes. The phenomenon appears at a rather large ratio of synchrotron frequency to the space charge tune shift, lower internal modes obtaining the stability at the larger ratio. Several modes of Gaussian bunch are considered in the paper, and their thresholds are estimated by comparison of the limiting cases.

\section{ACKNOWLEDGMENTS}

FNAL is operated by Fermi Research Alliance, LLC under Contract No. DE-AC02-07CH11395 with the United States Department of Energy.

[1] C. Pellegrini, Nuovo Cimento A 64, 447 (1969).

[2] M. Sands, SLAC Report No. TN-69-8, 1969.

[3] F. Sacherer, Report No. CERN-SI-BR-72-5, 1972.

[4] V. Balbekov, Zh. Tekh. Fiz. 46, 1470 (1976) [Sov. Phys. Tech. Phys. 21, 837 (1976)].

[5] V. Balbekov, Phys. Rev. ST Accel. Beams 12, 124402 (2009).

[6] V. Balbekov, Phys. Rev. ST Accel. Beams 14, 094401 (2011).

[7] L. Lasslett, V. Neil, and A. Sessler, Rev. Sci. Instrum. 36, 436 (1965).

[8] V. Balbekov and A. Kolomensky, At. Energ. 19, 1015 (1965); Plasma Phys. Controlled Nucl. Fusion Res. 8, 323 (1966)

[9] N. Dikansky and A. Skrinsky, At. Energ. 21, 176 (1966).

[10] A. Burov, Phys. Rev. ST Accel. Beams 12, 044202 (2009); 12, 109901 (2009). 